\title{
THE NONLINEAR EFFECTIVE ATTENUATION COEFFICIENT OF SPACEBORNE OCEANIC LIDAR SIGNAL
}

\author{
Qun Liu, Dong Liu*, Jian Bai, Xiaoyu Cui, Yudi Zhou, Peituo Xu, Zhipeng Liu, Xiaobin Wang \\ State Key Laboratory of Modern Optical Instrumentation, College of Optical Science and Engineering, \\ Zhejiang University, Hangzhou 310027, China \\ Email:*liudongopt@zju.edu.cn
}

\begin{abstract}
Multiple scattering is an inevitable effect in spaceborne oceanic lidar because of the large footprint size and the high optical density of seawater. The effective attenuation coefficient $k_{\text {lidar }}$ in oceanic lidar equation, which indicates the influence of the multiple scattering effect on the formation of lidar returns, is an important parameter in the retrieval of inherent optical properties (IOPs) of seawater. In this paper, the nonlinearity of $k_{\text {lidar }}$ and the relationships between $k_{\text {lidar }}$ and the IOPs of seawater are investigated by solving the radiative transfer equation with an improved semianalytic Monte Carlo model. $k_{\text {lidar }}$ is found to decrease exponentially with the increase of depth in homogeneous waters. $k_{\text {lidar }}$ is given as an exponential function of depth and IOPs of seawater. The results in this paper can help to have a better understanding of the multiple scattering effect of spaceborne lidar and improve the retrieval accuracy of the IOPs of seawater using spaceborne lidar.
\end{abstract}

\section{INTRODUCTION}

Lidars obtain depth-resolved return profiles by collecting backscattered signals from molecules and particles. It is essential to establish an accurate model of the radiative transfer process. The return signal of spaceborne oceanic lidar is strongly affected by multiple scattering due to the large footprint size and the high optical density of seawater [1]. To characterize the multiple-scattering radiative transfer process, an effective attenuation coefficient $k_{\text {lidar }}$ is introduced into the oceanic lidar equation. Gordon [2] found that the ratio of the radius of the spot on the sea surface viewed by the lidar receiver optics to the mean free path of photons in water is a significant parameter which is related to $k_{\text {lidar }}$. When this ratio is near zero, $k_{\text {lidar }}$ is given by the beam attenuation coefficient. If the ratio is greater than $5, k_{\text {lidar }}$ is given by the diffuse attenuation coefficient $K_{d}$. Phillips et al. [3] proposed a slightly different assumption that the upper and lower limits of $k_{\text {lidar }}$ is given by the beam attenuation coefficient and the absorption coefficient, respectively, depending on field of view (FOV). Collister et al. [4] exhibited the similarity between $k_{\text {lidar }}$ and the absorption coefficient under wide FOV using shipborne lidar measurements. The depth characteristics of $k_{\text {lidar }}$ are ignored in aforementioned studies. the values of $k_{\text {lidar }}$ at different depths in homogenous water are not constant, due to the multiple scattering effect $[5,6]$, especially near the ocean surface. This phenomenon leads to errors in the retrieval of optical properties, which further affects the assessment accuracy of the phytoplankton biomass and primary productivity. Thus, the depth characteristics of $k_{\text {lidar }}$ and the relationship between $k_{\text {lidar }}$ and the optical properties of seawater need to be studied in detail.

In this paper, the potential of using an improved semianalytic Monte Carlo radiative transfer simulation model (SALMON) to accurately identify the characteristics of both homogeneous and inhomogeneous waters is investigated. The use of SALMON is proposed by Poole et al. [5] and widely applied in oceanic and atmospheric lidars, therefore, it would be beneficial to develop an improved SALMON that can better simulate the inhomogeneous waters in the real oceans. We use this improved model to investigate the characteristics of lidar return profiles and the corresponding $k_{\text {lidar }}$. Analysis of the influence of the lidar system parameters on $k_{\text {lidar }}$ and the depth characteristic of $k_{\text {lidar }}$ for homogenous water are also inclueded. In addition, an exact relationship between the depth-resolved $k_{\text {lidar }}$ and IOPs of seawater is derived based on the statistical properties of case 1 waters. 


\section{METHODOLOGY}

\subsection{Lidar return signals}

The schematic diagram of the theoretical model of laser backscatter from water is illustrated in Fig. 1. The laser pulse transmits downward towards the flat horizontal water surface at the height of $H$. The photons are scattered and absorbed by the molecules and particles in seawater, and the backscattered photons are received by the detector forming the lidar return signal, which can be described by the lidar equation $[7,8]$ :

$$
\begin{array}{r}
P(z)=P_{0} M \frac{v \tau}{2 n} T_{\text {atm }}^{2} T_{\text {suff }}^{2} \frac{A}{(n H+z)^{2}} \times \\
\beta_{\pi}(z) \exp \left(-2 \int_{0}^{z} k_{\text {lidar }}\left(z^{\prime}\right) d z^{\prime}\right)
\end{array}
$$

where $P(z)$ is the power received from a depth of $z, P_{0}$ is the transmitted pulse power, $M$ is the lidar instrument constant, $v$ is the speed of light in vacuum, $\tau$ is the temporal pulse length, $v t /(2 n)$ is the depth resolution in water, $T_{a t m}$ is the transmission through the atmosphere, $T_{\text {surf }}$ is the Fresnel transmittance of the air-sea surface, $A$ is the receiver area, $n$ is the refractive index of sea water and $\beta_{\pi}$ is the volume scattering coefficient at a scattering angle of $\pi \mathrm{rad}$.

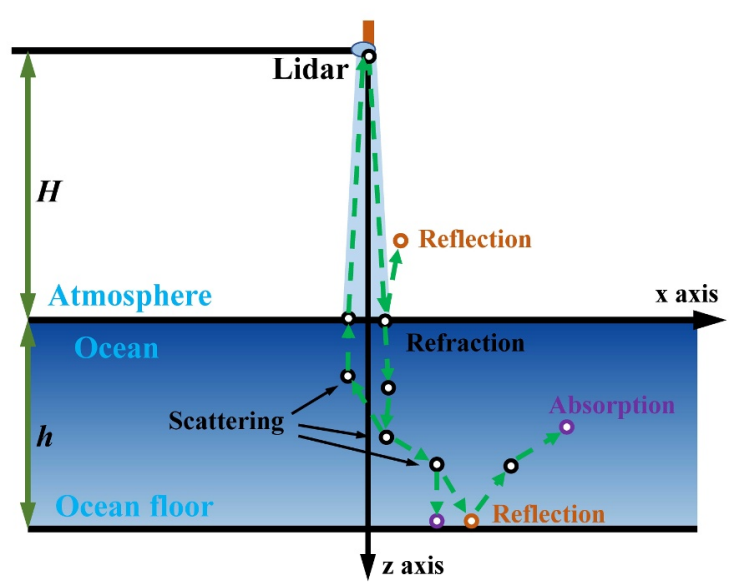

Figure 1. The schematic diagram of spaceborne lidar system.

Rewriting Eq.(1), we have the $P_{\text {norm }}(z)$ expressed by

$$
\begin{aligned}
P_{\text {norm }}(z) & =\frac{P(z) *(n H+z)^{2}}{P_{0} M(v \tau / 2 n) T_{\text {atm }}^{2} T_{\text {surf }}^{2} A \beta_{\pi}(z)} \\
& =\exp \left(-2 \int_{0}^{z} k_{\text {lidar }}\left(z^{\prime}\right) d z^{\prime}\right)
\end{aligned}
$$

Thus, the effective attenuation coefficient can be written as

$$
k_{\text {lidar }}(z)=-\frac{1}{2} \frac{d}{d z} \ln \left(P(z)_{\text {norm }}\right)
$$

$k_{\text {lidar }}$ is an important parameter for retrieving optical properties of seawater. The IOPs considered here include the scattering coefficient $b(\lambda) \quad\left(\mathrm{m}^{-1}\right)$, the absorption coefficient $a(\lambda)\left(\mathrm{m}^{-1}\right)$, and the beam attenuation coefficient $c(\lambda)\left(\mathrm{m}^{-1}\right)$.

\subsection{An improved semianalytic Monte Carlo radiative transfer simulation model}

Monte Carlo method is a numerical method of solving the radiative transfer. In 1981, Poole et al. [5] proposed a semianalytic Monte Carlo radiative transfer simulation model (SALMON) for atmosphere lidar, which is widely used in the current oceanic lidars. The improved SALMON proposed here is applicable for both homogeneous and inhomogeneous waters.

The SALMON model simulates the random collision and propagation in water of individual photons from the transmitted beam through a series of absorption and scattering until the photons move outside of the field of view of the lidar receiver or reach the boundaries of medium. The distance travelled before collision is the step size $(s)$ in dimensionless unit and defined as the integration of the attenuation coefficient over the photon pathway [9].. In the SALMON model, $s$ is calculated by $-\ln (R) / c$, where $R$ is a random value between 0 and 1 . The larger the attenuation coefficient $c$, the shorter distance the photon traveled before it is scattered or absorbed. For the stratified inhomogeneous waters, the IOPs are assigned separately for each layer. If the photon passes through more than one layer, $c$ will change along the pathway. Therefore, the actual step size in layer $i \pm 1$ could be calculated as $s_{i \pm 1}=\left(s_{i}-d_{i}\right) c_{i \pm 1} / c_{i}$, where $d$ is the geometrical distance along the ray in layer $i$ where the scattering took place. The sign 
indicates the photon propagates up (-) or down $(+)$. The photon travels until the initial step size has been consumed $\left(s_{i}<d_{i}\right)$. All the calculations in this paper are executed with more than $10^{11}$ photon packets to ensure a low level of standard deviation of the statistical estimates.

\section{RESULTS}

\subsection{Characteristics of lidar return signals for homogeneous case 1 waters}

The effective attenuation coefficient $k_{\text {lidar }}$ in lidar equation is the reflection of the IOPs of seawater. Determining the value of $k_{\text {lidar }}$ is beneficial to improve the retrieve accuracy of IOPs from lidar equation. In terms of the orbit altitude of $400 \mathrm{~km}$, the characteristics of lidar return signals from homogeneous case 1 water with various chlorophyll concentrations are studied. In these simulations, the divergence angle of the laser beam is assumed to be $0.1 \mathrm{mrad}$ and the diameter of the telescope is $1.5 \mathrm{~m}$ [10]. The ocean is assumed to have a depth of $50 \mathrm{~m}$ with a black bottom and the depth resolution is set to be $0.3 \mathrm{~m}$. The normalized lidar return signal power and the associated effective attenuation coefficients of seven groups of seawaters with [Chl] between 0 to $2 \mathrm{mg} / \mathrm{m}^{3}$, which covers most open ocean and productive coastal environment, are provided in Fig. 2. The normalized lidar return profiles in Fig. 2(a) shows that the linearity of the lidar return signal decreases with the increase of [Chl]. For seawaters with high [Chl], $k_{\text {lidar }}$ is not constant even if the IOPs are uniform in the vertical direction [Fig. 2(b)]. $k_{\text {lidar }}$ decreases with the increase of depth at first, then it becomes more stable. The full range of the nonlinearity in $k_{\text {lidar }}$ depends on the sensitivity of the receiving system. If this nonlinearity in $k_{\text {lidar }}$ is ignored in homogeneous waters, it may cause error in the retrieval of lidar signal.

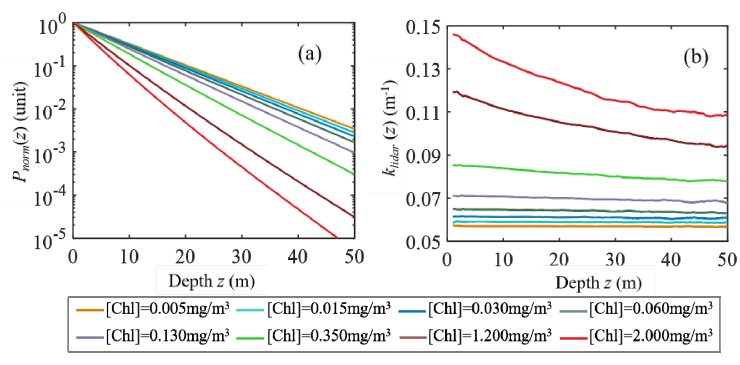

Figure 2. (a) Depth profiles of the normalized lidar return signals $P_{n o r m}(z)$ and (b) the corresponding effective attenuation coefficients $k_{\text {lidar }}(z)$ for [Chl] between 0 to 2 $\mathrm{mg} / \mathrm{m}^{3}$.

The nonuniformity of $k_{\text {lidar }}$ caused by multiple scattering is inevitable and it increases the uncertainty of the retrieval.

\subsection{The relationships between the klidar(z) and IOPs of water}

Based on the results shown in Fig. 3(b), an exponential formula is proposed to describe the depth characteristic of $k_{\text {lidar }}$, which is given by

$$
k_{\text {lidar }}(z)=m \times \exp (-n \times z)+p
$$

The slope and the asymptotic value of $k_{\text {lidar }}$ in Eq. (4) are determined by $m, n$ and $p$, respectively. Moreover, $k_{\text {lidar }}$ involves the IOPs of seawater, indicating these three parameters are related to the IOPs of seawater.

The influence of the IOPs on the qualitative behavior of $k_{\text {lidar }}$ is analyzed using a series of calculations with one variable at a time (Fig. 3). Fig. 3(a) shows that the curves of $k_{\text {lidar }}$ for different $\omega_{0}$ (with $b=0.2 \mathrm{~m}^{-1}$ ) are parallel to each other, which means that the absorption coefficient $a$ only affects the parameter $p$. Fig. 3(b) shows that the scattering coefficient $b$ influences both the slope and the asymptotic value of $k_{\text {lidar }}$. Fig. 3(c) shows that the phase function mainly affects the slope of the $k_{\text {lidar }}$ but has little influence on the asymptotic value of $k_{\text {lidar }}$. The dependence of $k_{\text {lidar }}$ on the phase function and $b$ can be summarized by a backscattering coefficient $b_{b}$, which is obtained by integrating the phase function in the range from $\pi / 2$ to $\pi$. 

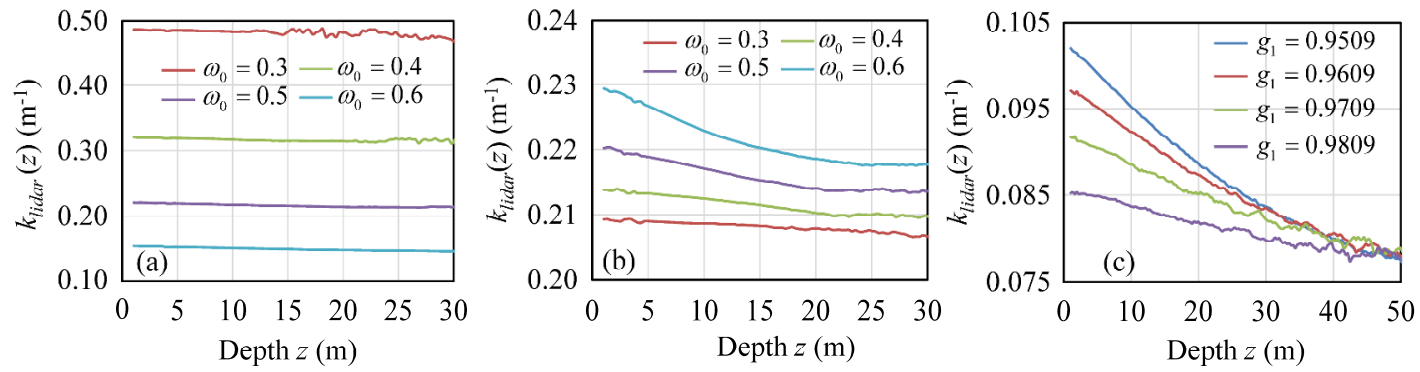

Figure 3. The influence of IOPs on $k_{\text {lidar }}$ for (a) $b=0.2 \mathrm{~m}^{-1}$ and $\omega_{0}=0.3(0.1) 0.6$, (b) $a=0.2 \mathrm{~m}^{-1}$ and $\omega_{0}=0.3(0.1) 0.6$, and (c) $[\mathrm{Chl}]=0.35 \mathrm{mg} / \mathrm{m}^{3}$ and $g_{l}=0.9509(0.01) 0.9809$.

In summary, the relationships among the parameters $m, n, p$ and IOPs can be derived. Both $m$ and $n$ show a linear relation with $b_{b}$, and $p$ is a linear function of $a$ and $b_{b}$. The linear functions can be expressed by

$$
\begin{aligned}
m & =4.8907 b_{b}-0.0004 \\
n & =4.2506 b_{b}-0.0055 \\
p & =a+0.3582 b_{b}-0.0042
\end{aligned}
$$

\section{CONCLUSIONS}

Multiple scattering effect on the spaceborne oceanic lidar returns is investigated with an improved SALMON, which includes a Gaussian beam emission model and is applicable for both homogeneous and inhomogeneous waters. The effective attenuation coefficient decays exponentially with the increase of depth in water according to the simulations of lidar return signal from case 1 waters, because high order scattering signals increase with depth and predominate the total signal at shallow depth. An exponential function is proposed to describe the depth characteristic of $k_{\text {lidar }}$ and the parameters in this exponential function is related to IOPs. The exact function of $k_{\text {lidar }}$, depth and the IOPs of seawater is given and examined using real ocean waters simulated by a model of inhomogeneous water.

\section{ACKNOWLEDGEMENTS}

National Key Research and Development Program of China (2016YFC1400900, 2016YFC0200700); National Natural Science Foundation of China (41775023, 61475141).

\section{REFERENCES}

[1] G. M. Krekov, M. M. Krekova, and V. S. Shamanaev, "Laser sensing of a subsurface oceanic layer. I. Effect of the atmosphere andwind-driven sea waves," Appl Opt 37, 1589-1595 (1998).
[2] H. R. Gordon, "Interpretation of airborne oceanic lidar: effects of multiple scattering," Applied Optics 21, 2996-3001 (1982).

[3] D. Phillips and B. Koerber, "A Theoretical Study of an Airborne Laser Technique for determining Sea Water Turbidity," Australian Journal of Physics 37, 75 (1984).

[4] B. L. Collister, R. C. Zimmerman, C. I. Sukenik, V. J. Hill, and W. M. Balch, "Remote sensing of optical characteristics and particle distributions of the upper ocean using shipboard lidar," Remote Sensing of Environment 215, 85-96 (2018).

[5] R. E. Walker and J. W. Mclean, "Lidar equations for turbid media with pulse stretching," Applied Optics 38, 2384-2397 (1999).

[6] V. I. Feygels, "Mathematical modeling of input signals for oceanographic lidar systems," Proc Spie 5155, 30-39 (2003).

[7] C. A. Hostetler, M. J. Behrenfeld, Y. Hu, J. W. Hair, and J. A. Schulien, "Spaceborne Lidar in the Study of Marine Systems," Annual Review of Marine Science 10(2017).

[8] J. H. Churnside, "Review of profiling oceanographic lidar," Optical Engineering 53, 051405 (2014).

[9] L. Wang, S. L. Jacques, and L. Zheng, MCMLMonte Carlo modeling of light transport in multilayered tissues (Springer US, 1995), pp. 131-146.

[10] D. M. Winker, M. A. Vaughan, A. Omar, Y. Hu, K. A. Powell, Z. Liu, W. H. Hunt, and S. A. Young, "Overview of the CALIPSO Mission and CALIOP Data Processing Algorithms," Journal of Atmospheric \& Oceanic Technology 26, 2310-2323 (2009). 